\title{
Dermoid cyst of the deep lobe of the parotid gland
}

\author{
Oscé Hanne ${ }^{1}$ (D), Vander Poorten Vincent ${ }^{1,2}$ (D) Hermans Robert3, Hens Greet ${ }^{1,4}$ (D) \\ 1Department of Otorhinolaryngology, Head and Neck Surgery, University Hospitals Leuven, Leuven, Belgium \\ 2Department of Oncology, section Head and Neck Oncology, KU Leuven, Leuven, Belgium \\ ${ }^{3}$ Department of Radiology, University Hospitals Leuven, Leuven, Belgium \\ ${ }^{4}$ Department of Neurosciences, Experimental ORL, KU Leuven, Leuven, Belgium
}

Cite this article as: Oscé H, Vander Poorten V, Hermans R, Hens G. Dermoid cyst of the deep lobe of the parotid gland. B-ENT 2020; 16(2): 137-40.

\begin{abstract}
A mass in the parotid gland may cause a diagnostic dilemma for the clinician. Cystic infra-auricular masses in pediatric patients are most often suggestive of a branchial cleft cyst. Dermoid cysts have only rarely been encountered in the parotid gland. Here, we present a case of a 15 -year-old female patient with a cystic mass in the left infra-auricular area. Total parotidectomy under continuous facial nerve monitoring was performed. Dermoid cysts of the parotid gland are extremely rare and a diagnostic challenge, but they still should be kept in mind during the differential diagnosis of persistent enlargement of the parotid gland in pediatric patients as well. We underline the necessity of thoroughly examining suspected cysts using ultrasonography or magnetic resonance imaging.
\end{abstract}

Keywords: Congenital cyst, dermoid cyst, parotid gland

\section{Introduction}

A dermoid cyst (DC) is a closed, epithelium-lined cavity, containing both ectodermal and mesodermal elements (1). These benign tumors may contain differentiated tissue and structures such as hair follicles, teeth, and skin glands. Rare cases of malignant transformation have been reported for DCs in the floor of the mouth with a probability of $5 \%(2,3)$.

DCs are most frequently localized in the coccyx (44.5\%) and ovaries (42.1\%). Overall, $7 \%$ of all DCs are seen in the head and neck area. In the head and neck region, the orbit, the nose, and the floor of the mouth account for $80 \%$ of DCs (4). More than $50 \%$ of these head and neck DCs are diagnosed before the age of six. In approximately one third of these patients, the DC is already present at birth. However, DCs have only rarely been encountered in the parotid gland. The current literature reports on only 20 cases of intraparotid DCs $(5,6)$. We report a rare case of an intraparotid DC in a female pediatric patient.

\section{Case Presentation}

A 15-year-old female patient was referred to our clinic for evaluation of a swelling of the left infra-auricular area, which she first noted four months earlier. She reported persistent pain during one month, followed by some episodes of acute pain.
She denied fever. There was no history of difficulty in swallowing or hearing loss. She mentioned having suffered from an ear infection and sore throat four months ago. The patient was not taking any medication and was otherwise in good health. She had no medical history of trauma or surgery related to this region. On physical examination, a mobile, soft mass was palpable inferior to the left pinna and measured approximately 2 $\mathrm{cm}$. Facial nerve function was normal on both sides. Visual inspection of the mouth revealed symmetrical and normal-size tonsils. Anterior rhinoscopy and otomicroscopy were normal.

Ultrasonography (US) (Figure 1) was carried out three weeks before this consultation and showed a well-defined, round mass $(32 \times 27 \times 29 \mathrm{~mm})$, located close to the left parotid gland. Multiple thin reflections in the lesion and some internal proliferations were observed. The US showed no vascularity. Radiologically, the mass was considered as a cystic lesion suggestive of first branchial cleft cyst. Magnetic resonance imaging (MRI) of the neck (Figures 2 and 3) was obtained to further define the extent and nature of the lesion, allowing a better preoperative evaluation. It confirmed a cystic mass $(28 \times 28 \times 40 \mathrm{~mm})$ in the superficial and deep lobe of the left parotid gland with high protein content and containing multiple small papillary projections originating from the internal border of the capsule. Superiorly, the mass was abutting the parapharyngeal space and the stylomastoid foramen. There was a close relationship with

Corresponding Author: Greet Hens, greet.hens@uzleuven.be

Received: May 17, 2020 Accepted: December 8, 2020

Available online at www.b-ent.be

CC BY 4.0: Copyright@Author(s), "Content of this journal is licensed under a Creative Commons Attribution 4.0 International License." 
the cartilaginous part of the external auditory canal; however no infiltration into the surrounding tissue was seen. Pathological lymph nodes were not detected. T2-weighted images demonstrated a high signal intensity. The presumed diagnosis was a branchial cleft cyst. However, based on the papillary projections, a cystic tumor could not be excluded. Fine-needle aspiration cytology (FNAC) was not performed.

The patient underwent a total parotidectomy (Figure 3). During surgery, a deformity of the cartilaginous part of the external auditory canal was visualized. Some evacuation of fluid from the cyst was seen. The superficial and the deep lobe of the left parotid gland containing the tumor were resected. The facial nerve branches were spared under continuous facial nerve monitoring. Postoperatively, the patient did not show any facial nerve impairment, and the further follow-up period was uneventful.

Pathological examination of the resection specimen demonstrated a cystic structure lined by stratified squamous epithelium with a maximal diameter of $23 \mathrm{~mm}$. The cyst wall was partially ruptured. The remaining parotid gland was otherwise histologically normal. The histology confirmed the diagnosis of a DC in the parotid gland. Informed consent of the parents was obtained for publication of the case.

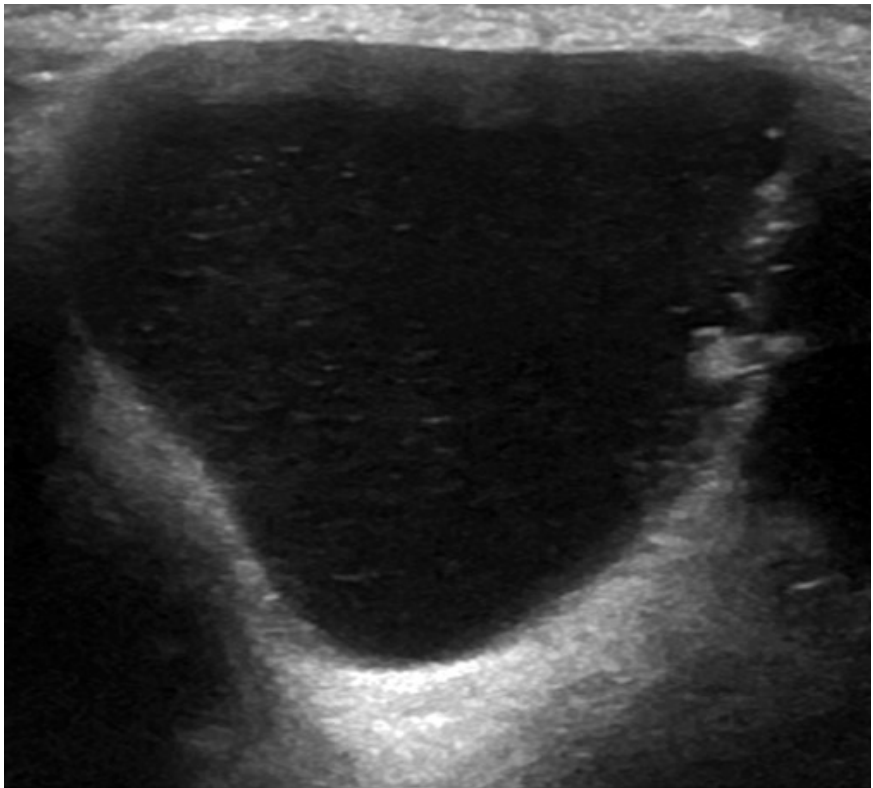

Figure 1. Ultrasonography of the left infra-auricular area showing a well-defined, homogenic, round mass measuring $32 \times 27 \times 29 \mathrm{~mm}$.

\section{Discussion}

A mass in the parotid gland can be a diagnostic challenge for the clinician. Cystic lesions make up $2 \%$ to $5 \%$ of all parotid gland lesions. DCs in the parotid gland are extremely rare. To our knowledge, this case report presents the 21st case worldwide and only the third one in a child (5-7). DCs usually remain asymptomatic until growth causes cosmetic or pressure-induced problems, which was the case in this patient. The mean age of patients at the time of diagnosis of an intraparotid DC is 31 years, higher than that of patients with DCs at other localizations (5)

The differential diagnosis of a parotid swelling includes a first branchial cleft cyst, benign and malignant salivary gland tumors, benign mesenchymal tumors, lymphoepithelial cyst, or mucous retention cyst (8). Physical examination has no characteristic findings. An accurate preoperative diagnosis is challenging, and the definite diagnosis in reported cases has generally been made retrospectively. However, radiological imaging may contribute to the differential diagnosis. US imaging typically reveals a well-circumscribed cystic lesion with mixed or pseudosolid appearance. MRI shows the exact localization and relation to surrounding tissue and usually presents a cystic mass, hyperintense on T2-weighted images and hypointense on T1-weighted images. The preoperative importance and reliability of FNAC are controversial $(7,9)$. It may provide reliable information on cystic lesions if there are supporting radiologic findings (10). However, FNAC can also be misleading because some well-differentiated squamous cell carcinomas and malignant lesions showing squamous metaplasia may share benign looking cytologic features with DCs (11). In this case, an FNAC was not performed after considering the patient's age and the necessity of a total parotidectomy for tumor resection. The definite diagnosis should always be made by histological examination.

New and Erich (4) classified DCs into three groups: (1) congenital DCs of the teratoma type; (2) acquired DCs owing to the traumatic implantation of epithelium, such as during punction; and (3) congenital inclusion DCs. The last group was further subdivided into four subgroups of cysts according to their anatomical localization and embryogenesis: cysts of the (a) peri-orbital region; (b) nose; (c) floor of mouth and submental and submaxillary region; and (d) midventral and middorsal fusion line (suprasternal, suboccipital, thyroidal, lower lip, and palate region) (4). A classification of DCs in the parotid gland is still controversial owing to the rarity. In this case, there was no history of trauma or
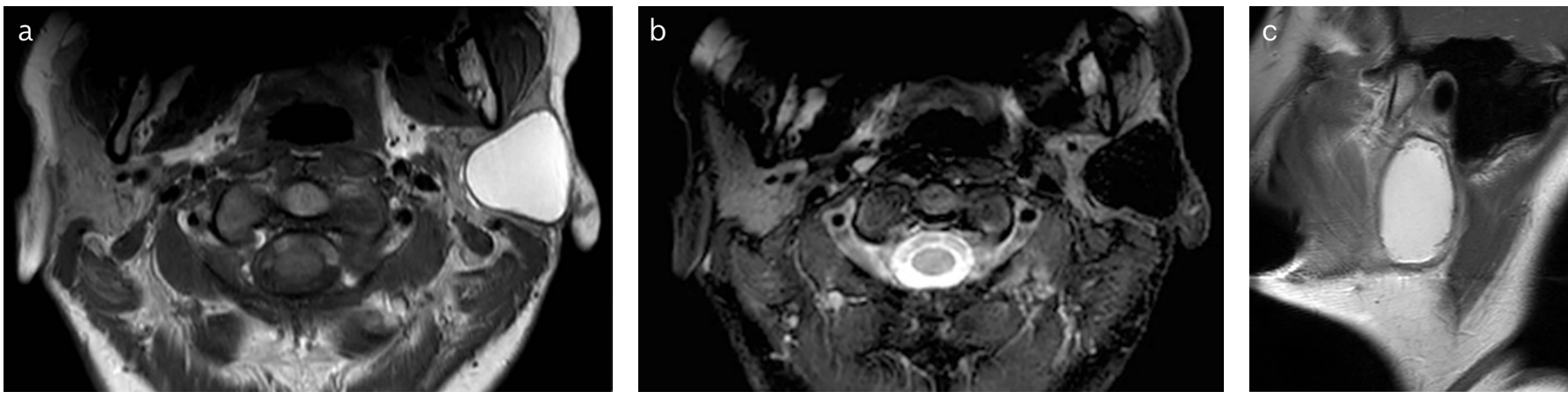

Figure 2. a-c. MRI in T2 sequence showing location of a well-defined intraparotid mass and exerting mass effect toward the left EAC. EAC, external auditory canal; MRI, magnetic resonance imaging $(a-c)$. 

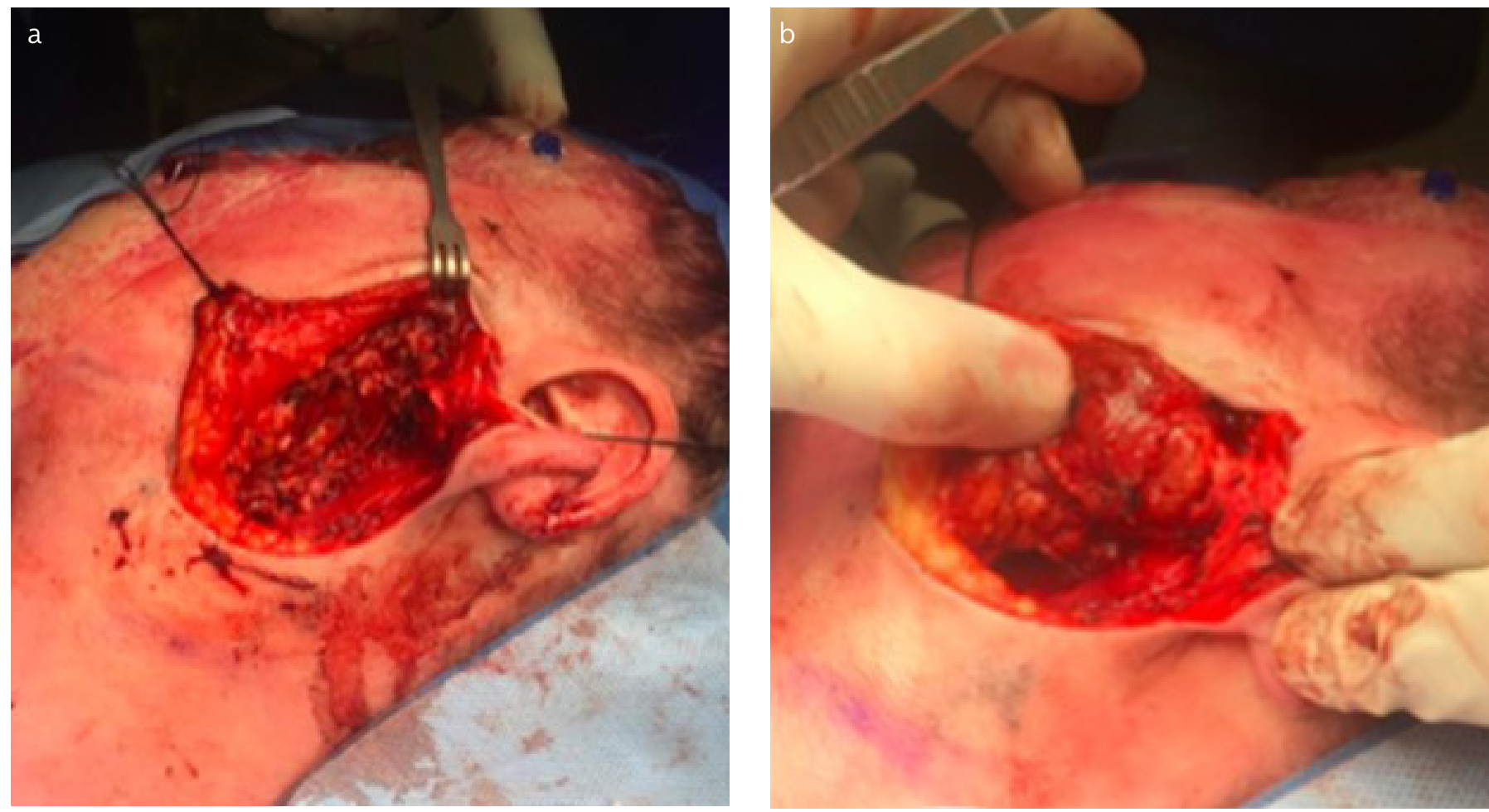

Figure 3. a, b. Intraoperative photographs showing anomaly of the cartilaginous part of the left EAC. EAC, external auditory canal (a, b).

prior surgery in the parotid area that could have led to traumatic implantation of epithelial cells. The young age of this patient also supports the congenital theory. Furthermore, the abnormal cartilaginous part of the external auditory canal could be caused by long-standing compression because of the cystic mass. Further research is necessary to determine to which category the DC of the parotid gland belongs.

Parotidectomy is the definite treatment for DCs in the parotid gland, and the surgical plan needs to be tailored to each case. Superficial parotidectomy is the standard procedure for lesions in the superficial lobe. Total parotidectomy is recommended for DCs located in the deep lobe of the parotid gland (1). Preservation of the facial nerve by meticulous dissection is a key issue in these surgeries and can be facilitated by intraoperative neuromonitoring.

In this case report, we aim to highlight the possibility of a DC of the parotid gland in young patients initially presenting with an infra-auricular cystic mass mimicking the more common first branchial cleft cyst.

Dermoid cysts are benign tumors. DCs are often asymptomatic, although they may cause pressure-induced or cosmetic problems. Accurate preoperative diagnosis is challenging; however, proper preoperative examination using US or MRI is important. Complete surgical excision by facial nerve-sparing superficial or total parotidectomy is suggested to avoid recurrence. The definite diagnosis can only be confirmed by histopathological examination.

Informed Consent: Verbal informed consent was obtained from the parents of the patient who agreed to take part in the study.
Peer-review: Externally peer-reviewed.

Author Contributions: Supervision - H.G.; Design - O.H., H.G.i Supervision - H.G.; Literature Search - O.H.; Writing Manuscript - O.H., H.G.; Critical Review - H.G., V.V., H.R.

Conflict of Interest: The authors have no conflict of interest to declare.

Financial Disclosure: The authors declared that this study has received no financial support.

\section{References}

1. Rosen D, Wirtschafter A, Rao VM, Wilcox Jr TO. Dermoid cyst of the lateral neck: A case report and literature review. Ear Nose Throat J 1998; 77: 125. [Crossref]

2. Gold BD, Sheinkopf DE, Levy B. Dermoid, epidermoid, and teratomatous cysts of the tongue and the floor of the mouth. J Oral Surg 1974; 32: 107-11.

3. Devine JC, Jones DC. Carcinomatous transformation of a sublingual dermoid cyst: a case report. Int J Oral Maxillofac Surg 2000; 29: 126-7. [Crossref]

4. New GB, Erich JB. Dermoid cysts of the head and neck. Surg Gynecol Obstet 1937; 65: 48.

5. Yigit N, Karslioglu Y, Yildizoglu U, Karakoc O. Dermoid cyst of the parotid gland: Report of a rare entity with literature review. Head Neck Pathol 2015; 9: 286-92. [Crossref]

6. Glaas MF, Schipper J, Kajasi N, Albrecht A. The youngest reported and successfully treated patient with a dermoid cyst of the parotid gland: A rare pediatric case. Case Rep Otolaryngol 2017; 2017: 4187030. [Crossref]

7. Tas A, Yagiz R, Altaner S, Karasalihoglu AR. Dermoid cyst of the parotid gland: first pediatric case. Int J Pediatr Otorhinolaryngol 2010; 74: 216-7. [Crossref]

8. Saylam G, Tanrikulu S, Dursun E, Iriz A, Eryilmaz A. A mass at fat density in the parotid gland: dermoid cyst or lipoma. B-ENT 2009; 5: 43-5. 
9. Baschinsky D, Hameed A, Keyhani-Rofagha S. Fine- needle aspiration cytological features of dermoid cyst of the parotid gland: A report of two cases. Diagn Cytopathol 1999; 20: 387-8. [Crossref]
10. Islam S, Hoffman GR. Parotid dermoid cyst: a rare entity. J Laryngol Otol 2009; 123123: e7. [Crossref]

11. Birsan C, Raza A, Cobb C. Parotid dermoid cyst: A case report. Int J Surg Pathol 2013; 21: 197-201.[Crossref] 